\title{
Configurations d'écoulement gaz-liquide
}

\author{
Gas/liquid flow configurations
}

Jacques Bonnin* et Jean-Michel Fitremann**

Dans le cadre des activités du Groupe de Travail "Ecoulements Polyphasiques" de la Société Hydrotechnique de France, un certain nombre de réflexions sur le problème des configurations d'écoulements diphasiques gaz-liquide sont présentées en vue de guider d'éventuels travaux spécifiques sur ce sujet. Les auteurs ont animé ces discussions avec la participation de plusieurs industriels et présentent ce résumé des travaux.

Les études théoriques et expérimentales des écoulements diphasiques ont une application immédiate au fonctionnement des réacteurs nucléaires, à l'extraction et au transport des hydrocarbures, au génie chimique, etc. Les écoulements présentent des aspects géométriques des interfaces variés selon les conditions de l'écoulement et la nature des fluides. La description précise de ces configurations et de leurs domaines d'existence est difficile, mais nécessaire pour la prévision et le calcul des écoulements.

\section{Classifications proposées des configurations d'écoulement}

Une liste de configurations de complexité croissante a été indiquée par $M$. Bonnin [1] sur des bases topologiques. Dans le même ordre d'idée M. Fitremann [2] utilise une méthode combinatoire pour décrire les diverses configurations à partir de géométries élémentaires. Cette méthode peut se révéler intéressante pour fabriquer des modèles élémentaires et obtenir la similitude par décomposition de la géométrie de l'écoulement en configurations plus simples. Voir figures 1 et 2 extraites de [1].

(*) E.D.F. Direction des Etudes et Recherches, 6, Quai Watier, 78400 CHATOU

(**) D.I.P. Laboratoire de Mécanique Expérimentale des Fluides, Bât. 502, 91405 ORSAY.

\section{Analyse paramétrique et similitude}

Les parametres de similitude indépendants essentiels sont décrits dans [1] et [2] pour les cas d'un écoulement en canal avec transfert de masse et sont au nombre de 8 . Des raisons physiques tenant aux cas d'application les plus usuels permettent de penser qu'une réduction est possible, typiquement de 8 à 4 ou 5 selon les cas. II convient toutefois de remarquer que cette réduction ne peut pas être universelle (ex : écoulements annulaires à gouttelettes vertical : 6 paramètres dans le modèle décrit dans [2]). De plus, la coalescence joue un rôle important dans l'établissement de certaines configurations (transition bulles $\rightarrow$ poches par exemple) et un paramètre supplémentaire décrivant ce phénomène est indipensable.

\section{Méthodes d'investigation}

A l'aide de capteurs sensibles à un domaine ponctuel ou étendu de l'espace (sur une corde de la section, une section ou un volume d'écoulement), les chances d'identifier à coup sûr une configuration donnée varient selon l'emplacement du ou des capteurs, leur nombre et l'inclinaison de la conduite. On a dressé un tableau des possibilités des différentes méthodes de diagnostic en donnant une côte d'intérêt pour chaque méthode et type d'écoulement à détecter.

Le tableau I donne les possibilités de détection d'une configuration en fonction du type de méthode choisi et surtout de l'étendue du volume de mesure. Le tableau II donne des renseignements complémentaires pour des méthodes d'intercorrélation au sens large.

Pour le détail des méthodes de mesure, on pourra consulter le travail du séminaire "Métrologie des Ecou- 


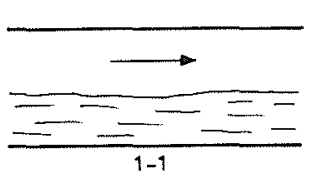

stratifié (2)

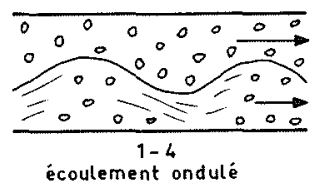

dispersé avec bulles (3)
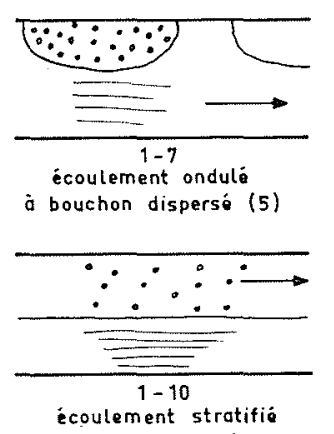

dispersé (8)

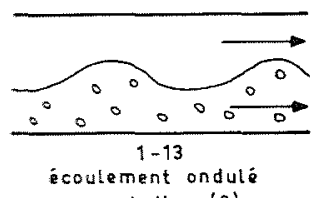

arec butles (g)

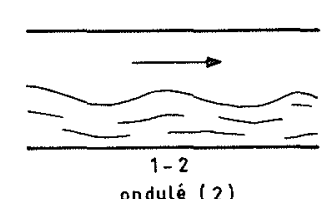

ondulé (2)

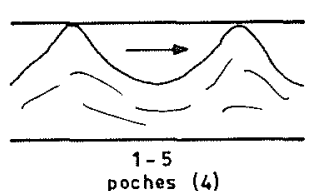

poches (4)

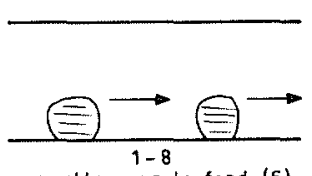

$1-8$
gouttes sur lo fond (6)

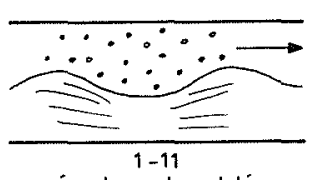

coulement ondul

dispersé (8)

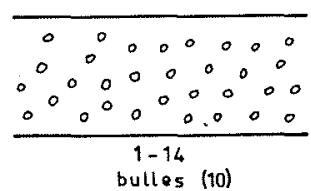

bulles (10)

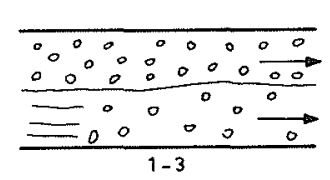

écoulement stratifie dispersé avec bulles (3)
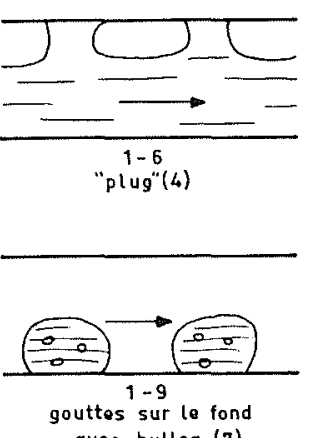

avec bulles (7)

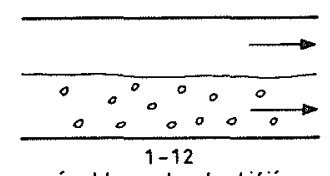

collement stratifio

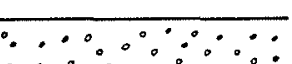

$\because \therefore \therefore \therefore \therefore \therefore \therefore$

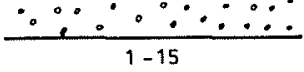

brovillard (16)

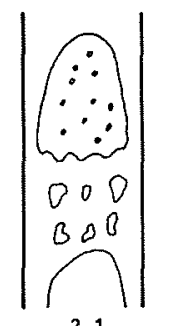

2-1

bouchous disperses (1)

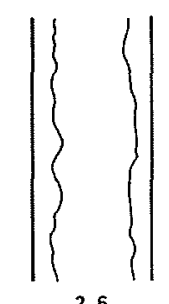

2-6

annulaire (12)

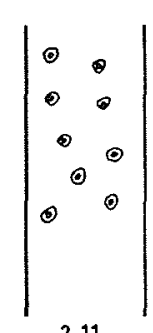

2-11

brovillard aéré $(7)$

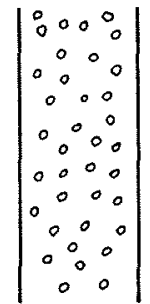

$2-2$

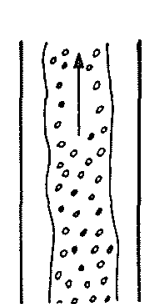

$2-7$
ecoulement

annulaire
dispersé (13)

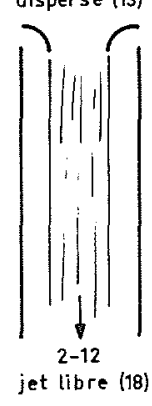

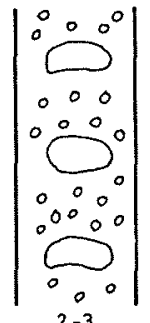

$2-3$

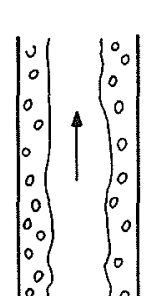

in

$2-8$
écoulement

annulaire
ovec bulles (14)

roc

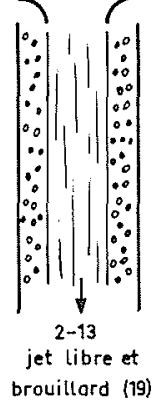

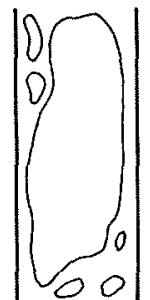

$2-4$

"churn"(10)

("churnotique")

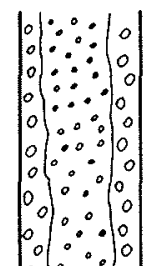

2-9

ment annulaire disperse
avec bulles (15) C $r$

$\mid \begin{array}{lll}0 & 0 \\ 0 & 0 \\ 0 & 0 \\ 0 & 0 \\ 0 & 0 \\ 0 & 0 \\ 0 & 0 \\ 0 & 0 \\ 0 & 0 \\ 1 & 0 \\ 2-14 & \\ \text { jet libre } & \end{array}$
jet libre
oéré $(20)$

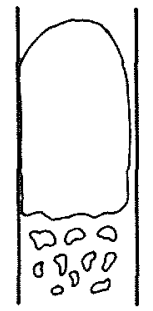

$2-5$

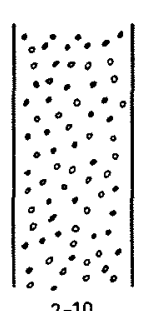

2-10

brovillard (16)

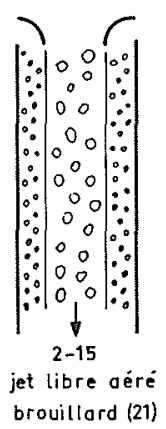

brouillard (21)
Les numéros entre parenthèses renvoient à ceux du tableou I

Figure 1 - Configuration d'écoulements diphasiques liquide-gaz
Les numéros entre parenthèses renvoient à ceux du tableau I

Figure 2-Configuration d'écoulements diphasiques liquide-gaz (suite) 


\begin{tabular}{|c|c|c|c|c|c|c|c|c|}
\hline $\begin{array}{l}\text { Type de } \\
\text { mesure }\end{array}$ & $\begin{array}{l}\text { Métho } \\
\text { proc }\end{array}$ & $\begin{array}{l}\text { de ou } \\
\text { édé }\end{array}$ & $\begin{array}{c}\text { Caractéristiques } \\
\text { physiques } \\
\text { principales }\end{array}$ & $\begin{array}{l}\text { Grandeur } \\
\text { mesurée }\end{array}$ & $\begin{array}{l}\text { Cas d'application } \\
\text { possible de la } \\
\text { méthode }\end{array}$ & $\begin{array}{l}\text { Configurations } \\
\text { discernables }\end{array}$ & Remarques & Cote \\
\hline \multirow{4}{*}{$\begin{array}{l}\text { Intégrales de } \\
\text { volume } \\
\text { et } \\
\text { assimilées } \\
\vartheta \text { ou } \mathcal{S} \times \mathscr{C}\end{array}$} & \multicolumn{2}{|c|}{ Vannes rapides } & $\begin{array}{l}\tau \sim 1 \mathrm{~ms} \\
L \gtrsim D\end{array}$ & $\begin{array}{l}\text { Taux de présence } \\
\text { moven }\end{array}$ & $\begin{array}{l}\text { Detection de } \\
\text { bouchons pos- } \\
\text { sible } \\
\text { Vertical, Horizon- } \\
\text { tal }\end{array}$ & Bouchons $\leftrightarrow$ Homogène & Méthode lourde & $*$ \\
\hline & \multicolumn{2}{|c|}{$\begin{array}{l}\text { Débimétrie } \\
\text { (Moulinet) }\end{array}$} & variées & vitesse ou débit & $\begin{array}{l}\text { Vertical, } \\
\text { (Horizontal ?) }\end{array}$ & $\begin{array}{l}\text { Bouchons } \leftrightarrow \text { bulles } \\
\text { Bouchon } \leftrightarrow \text { stratifié }\end{array}$ & $\begin{array}{l}\text { Essai à faire en } \\
\text { régimes bouchon } \\
\text { (section totale ou } \\
\text { partielle) }\end{array}$ & * \\
\hline & \multicolumn{2}{|c|}{$\begin{array}{l}\text { Débimétrie } \\
\text { Electromagnétique }\end{array}$} & $L \sim D$ & $\sim$ débit & $\begin{array}{l}\text { Vertical } \\
\text { Horizontal }\end{array}$ & $\begin{array}{l}\text { Bouchons } \leftrightarrow \text { bulles } \\
\text { Bouchons } \leftrightarrow \text { annulaire } \\
\text { Bouchons } \leftrightarrow \text { Stratifié } \\
\leftrightarrow \text { vagues }(\lambda \sim D)\end{array}$ & & ** \\
\hline & \multicolumn{2}{|c|}{ Célérité du "son" } & $L>D$ & $\begin{array}{l}\text { intégrale } \\
\text { pondérée }\end{array}$ & $\begin{array}{l}\text { Vertical } \\
\text { Horizontal }\end{array}$ & $\begin{array}{l}\text { Stratifié } \leftrightarrow \text { Bulles } \\
\leftrightarrow \text { Bouchons (?) }\end{array}$ & & $\stackrel{*}{*}$ \\
\hline \multirow{5}{*}{$\begin{array}{l}\text { Intégrales } \\
\text { de } \\
\text { surface } \\
\text { (section) } \\
\quad \&\end{array}$} & \multirow{4}{*}{$\begin{array}{l}\text { Absorp- } \\
\text { tion }\end{array}$} & $x$ & $\begin{array}{c}\tau>10 \mu \text { s tous } \\
\text { liquides }\end{array}$ & \multirow{2}{*}{$\begin{array}{l}\text { taux de présence } \\
\text { moyen sur la } \\
\text { section et sur le } \\
\text { temps } \tau\end{array}$} & \multirow{2}{*}{$\begin{array}{l}\text { Vertical } \\
\text { Horizontal }\end{array}$} & \multirow{2}{*}{$\begin{array}{l}\text { Poches } \leftrightarrow \text { Bulles } \\
\text { (faible épaisseur) } \\
\text { Bouchons } \leftrightarrow \text { Film } \leftrightarrow \\
\text { Film ondulé }\end{array}$} & \multirow{2}{*}{$\begin{array}{l}\text { Limites } \tau \\
\text { selon la puissance } \\
\text { de source. Diffi- } \\
\text { cultés en paroi } \\
\text { épaisse. }\end{array}$} & \multirow[t]{2}{*}{ ** } \\
\hline & & $\stackrel{\gamma}{\beta, n}$ & $\begin{array}{l}\tau \text { en général } \\
\text { élevé }\end{array}$ & & & & & \\
\hline & & $\begin{array}{l}\text { micro- } \\
\text { ondes }\end{array}$ & & & : & & à essayer & $?$ \\
\hline & & optique & & & Horizontal & Stratifié & & $*$ \\
\hline & \multicolumn{2}{|c|}{$\begin{array}{l}\text { Diffusion } \\
\text { Diffraction }\end{array}$} & & & & Gouttelettes & Eau, Vapeur & ${ }^{*}$ \\
\hline $\begin{array}{l}\text { Intégrales } \\
\text { Linéaires } \\
\qquad \mathcal{e}\end{array}$ & \multicolumn{2}{|c|}{ Mêmes methodes } & & & $\begin{array}{l}\text { Vertical } \\
\text { Horizontal }\end{array}$ & & $\begin{array}{l}=\text { une ou deux } \\
\text { cordes orthogo- } \\
\text { nales } \\
\text { choix judicieux } \\
\text { des cordes }\end{array}$ & ** \\
\hline \multirow{4}{*}{$\begin{array}{l}\text { Sondes } \\
\text { Locales } \\
\delta \vartheta\end{array}$} & \multicolumn{2}{|c|}{$\begin{array}{l}\text { Présence de } \\
\text { phase }\end{array}$} & $L \sim 25 \mu \mathrm{m}$ & $\begin{array}{l}\text { Présence } \\
\text { locale instantanée }\end{array}$ & $\begin{array}{l}\text { Vertical } \\
\text { Horizontal }\end{array}$ & $\begin{aligned} \text { Buttes } \leftrightarrow \text { Bouchons } \\
\leftrightarrow \text { Films } \leftrightarrow \text { Gouttes } \\
\text { (grosses) }\end{aligned}$ & $\begin{array}{l}\text { Associé à une } \\
\text { mesure de vitesse: } \\
\text { spectre de di- } \\
\text { mensions }\end{array}$ & $* * *$ \\
\hline & \multicolumn{2}{|c|}{ Température } & $L \sim 100 \mu \mathrm{m}$ & $\begin{array}{l}\text { Température } \\
\text { locale instantanee }\end{array}$ & $\begin{array}{l}\text { Vertical } \\
\text { Horizontal }\end{array}$ & & Eau, Vapeur & $*$ \\
\hline & \multicolumn{2}{|c|}{$\begin{array}{l}\text { Absorption/Fais- } \\
\text { ceaux croisés }\end{array}$} & $L \sim 50 \mu \mathrm{m}$ & $\begin{array}{l}\text { Densité locale } \\
\text { instantanée }\end{array}$ & $\begin{array}{l}\text { Vertical, } \\
\text { Horizontal }\end{array}$ & Microgouttes & $\begin{array}{l}\text { Eau, Vapeur. } \\
\text { Se rapproche } \\
\text { d'intercorrélation } \\
\text { de sondes type }\end{array}$ & . \\
\hline & \multicolumn{2}{|c|}{$\begin{array}{l}\text { Sonde électro- } \\
\text { magnétique }\end{array}$} & $L \sim$ qques $\mathrm{mm}$ & $\begin{array}{l}\sim \text { vitesse locale } \\
\text { instantanee }\end{array}$ & $\begin{array}{l}\text { Vertical, } \\
\text { Horizontal }\end{array}$ & $\begin{array}{l}\text { Bulles } \leftrightarrow \text { Bouchon } \leftrightarrow \\
\text { Stratifie } \leftrightarrow \text { Gouttes }\end{array}$ & & $* *$ \\
\hline \multirow{4}{*}{$\begin{array}{l}\text { Sondes } \\
\text { pariétales } \\
\qquad \delta\end{array}$} & \multicolumn{2}{|c|}{$\begin{array}{l}\text { Prise de pression } \\
\text { Capteur affleurant }\end{array}$} & $\left\{\begin{array}{l}L \sim 0.2 \mathrm{~mm} \\
\tau \sim 1 \mathrm{~ms} \\
L \sim 3 \mathrm{~mm}\end{array}\right.$ & $\begin{array}{l}\text { pression } \\
\text { à la paroi }\end{array}$ & $\begin{array}{l}\text { Vertical } \\
\text { Horizontal }\end{array}$ & $\begin{array}{l}\text { Bulles } \leftrightarrow \text { Bouchon } \\
\text { Bouchon } \leftrightarrow \text { Annulaire } \\
\text { Bouchons } \leftrightarrow \text { Stratifié }\end{array}$ & & ** \\
\hline & \multicolumn{2}{|c|}{$\begin{array}{l}\text { Présence de phase } \\
\text { pariétale }\end{array}$} & $L \sim 0.1 \mathrm{~mm}$ & $\begin{array}{l}\text { présence de } \\
\text { phase interne }\end{array}$ & $\begin{array}{l}\text { Vertical } \\
\text { Horizontal }\end{array}$ & $\begin{array}{l}\text { Films } \\
\text { Bouchons }\end{array}$ & $\begin{array}{l}\text { Sondes à la paroi } \\
\text { superieure }\end{array}$ & $* * *$ \\
\hline & \multicolumn{2}{|c|}{$\begin{array}{l}\text { Sonde électro- } \\
\text { magnétique }\end{array}$} & $L \sim$ qques $\mathrm{mm}$ & $\begin{array}{l}\text { Vitesse dans la } \\
\text { couche limite }\end{array}$ & $\begin{array}{l}\text { Vertical } \\
\text { Horizontal }\end{array}$ & $\begin{array}{l}\text { Films } \\
\text { Bouchons }\end{array}$ & & ** \\
\hline & \multicolumn{2}{|c|}{$\begin{array}{l}\text { Frottement } \\
\text { pariétal }\end{array}$} & $L \sim 0.1 \mathrm{~mm}$ & $\begin{array}{l}\text { Gradient de } \\
\text { vitesse a la paroi }\end{array}$ & $\begin{array}{l}\text { Vertical } \\
\text { Horizontal }\end{array}$ & $\begin{array}{l}\text { Bulles } \leftrightarrow \\
\text { Bouchons } \leftrightarrow \text { Films }\end{array}$ & $\begin{array}{l}\text { Distingue le sens } \\
\text { de f'écoulement }\end{array}$ & $* * *$ \\
\hline
\end{tabular}




\begin{tabular}{|c|c|c|c|c|}
\hline Tableau II & & \multicolumn{3}{|c|}{ Intérêt des mesures par corrélation spatiale ou temporelle de deux sondes } \\
\hline j & $\mathscr{S}$ & f & $\delta ?$ & $\delta$ \\
\hline$P \rightarrow$ & & \multicolumn{3}{|c|}{$\begin{array}{l}\text { * qualification possible de méthodes globales de recherche de configuration } \\
\text { dans certains cas }\end{array}$} \\
\hline \multirow[t]{4}{*}{$\mathscr{S} \rightarrow$} & $\begin{array}{l}\text { * étude des écoule- } \\
\text { ments a forte variation } \\
\text { axiale des propriétés } \\
* * \text { Holographie }\end{array}$ & $\begin{array}{l}\text { * qualification de cer- } \\
\text { taines cordes privilégiées } \\
\text { pour les méthodes par } \\
\text { absorption }\end{array}$ & $\begin{array}{l}\text { * qualification possible } \\
\text { de méthodes globales }\end{array}$ & \multirow{2}{*}{$\begin{array}{l}\text { * } \text { caractérisation de } \\
\text { la configuration a bou- } \\
\text { chons en écoulement } \\
\text { non-vertical }\end{array}$} \\
\hline & \multirow[t]{3}{*}{$\mathscr{L} \rightarrow$} & $\begin{array}{l}\text { *** choix de deux } \\
\text { cordes pour écoulements } \\
\text { non verticaux } \\
\text { *** méthode à deux } \\
\text { longueurs d'onde pour } \\
\text { analyser des écoule- } \\
\text { ments à bouchons }\end{array}$ & $\begin{array}{l}\text { ** éta lonnage de } \\
\text { sondes locales }\end{array}$ & \\
\hline & & \multirow[t]{2}{*}{$\delta \% \rightarrow$} & $\begin{array}{l}\text { ** granulométrie, } \\
\text { vitesse de transit des } \\
\text { interfaces }\end{array}$ & $\begin{array}{l}* \text { * caractérisation des } \\
\text { interfaces et de leur } \\
\text { géometrie possible } \\
{ }^{* *} \text { distinction entre } \\
\text { vagues et bouchons en } \\
\text { horizontal }\end{array}$ \\
\hline & & & $\delta \mathscr{S} \rightarrow$ & $\begin{array}{l}\text { ** vitesses de transit } \\
\text { des interfaces } \\
\text { * }^{*} \text { etudes des films } \\
\text { minces } \\
\text { * }^{*} \text { distinction de la } \\
\text { configuration bouchons } \\
\text { en horizontal }\end{array}$ \\
\hline
\end{tabular}

lements Diphasiques - S.H.F." décrit par ailleurs dans cette revue.

L'analyse de l'aspect géométrique d'une configuration complexe (bouchons et bulles, brouillard et vagues, etc.) ne peut se faire que par une analyse statistique des signaux de sondes. En conséquence, la limite entre deux configurations n'a pas un caractère purement déterministe et doit (dans la plupart des cas) être définie par une notion de probabilité d'obtenir telle ou telle valeur géométrique d'une dimension caractéristique des interfaces. Cette approche est indispensable dans toute étude de transition de configurations (ex. [3]).

\section{Domaines d'intérêt}

Dans le souci de limiter le volume des études à entreprendre tout en restant dans le domaine des applications les plus fréquentes, des intervalles de variation des paramètres physiques dimensionnels ont été proposés (cf. tableau III). Les investigateurs pourront dans un premier temps chercher a éliminer les groupements adimensionnels les moins influents et en déduire le domaine d'intérêt sous forme non-dimensionnelle.

\section{Considérations sur les installations}

La configuration se produisant dans une conduite peut être sensible aux conditions d'entrée-sortie. Un certain nombre de précautions sont donc indispensables pour assurer la validité des résultats et leur transposition éventuelle à d'autres installations. Une liste de recommandations a été établie afin que les conditions expérimentales soient fixées en vue de cette validation.

1 - Contrôler les effets d'entrée. L'établissement de conditions d'écoulement peu évolutives nécessite dans les meilleurs cas (vertical, haute pression) des longueurs de 100 diamètres au moins, dans les cas usuels (horizontal, vertical, basse pression) cette longueur peut atteindre 500 à 1000 diametres.

Il est nécessaire de contrôler l'évolution des propriétés en fonction de l'abscisse.

2 - Conditions d'injection. Pour diminuer les longueurs d'établissement, on a intérêt à choisir un mode d'injection adapté. Il est donc indispensable de disposer d'au moins un autre mode d'injection très différent afin de vérifier la sensibilité du régime ou de la transition au mode d'injection.

3 - Oscillations d'installation. Le découplage des conditions d'entrée-sortie est nécessaire pour obtenir les configurations se produisant dans des conditions "naturelles". On doit avoir soin de réguler les débits à l'injecteur de façon correcte (blocage sonique, fortes pertes de charge, régulation) et de minimiser les fluctuations des pertes de charge singulière dans les organes de sortie (séparateurs spéciaux pour écoulements à bouchons, décantation, etc ...).

4 - Propriétés des fluides. On doit s'efforcer si possible de contrôler l'effet de la contamination des inter- 


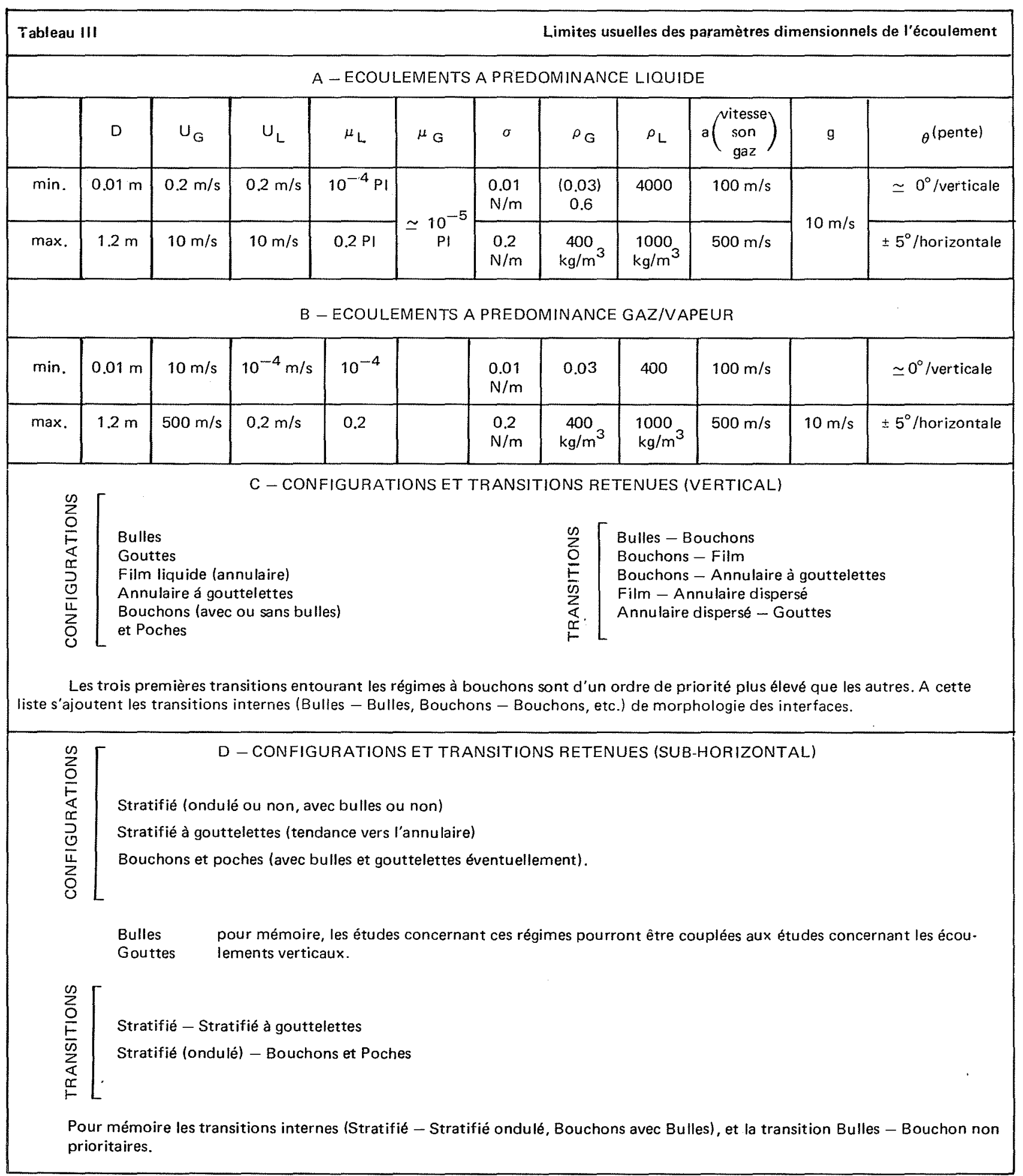

faces et de s'assurer de l'équilibre thermodynamique entre les phases.

5 - Métrologie

a) on consultera le groupe "Séminaire Métrologie" de la S.H.F - Groupe Ecoulements Polyphasiques en ce qui concerne les questions de sondes et d'interprétation des signaux,

b) voir les tableaux I et II en ce qui concerne l'adaptation entre méthodes de mesure et configurations à étudier, c) les transitions sont généralement progressives. I1 conviendra donc dans ce cas d'en faire une analyse statistique.

\section{Conclusions}

L'étude des configurations rencontrées dans une vaste plage de régimes d'écoulement dans des conduites circulaires en écoulement isotherme pour des pentes 
voisines de l'horizontal ou verticales présente un intérêt considérable. Le travail à faire est de grande ampleur eu égard

1 - au nombre élevé de paramètres indépendants

2 - à la nature statistique de la définition des transitions qui doit remplacer la définition "visuelle"

3 -à l'importance des dimensions nécessaires des installations compte tenu des effets d'établissement de régime.

Dans bien des cas, la notion de transition pourra varier selon les capteurs et les critères utilisés et il conviendra d'en faire une comparaison rigoureuse.

Les résultats d'une telle étude se présenteront sous la forme de cartes multidimensionnelles de configurations interprétées selon les grandeurs statistiques déduites de telle ou telle méthode. L'usage prévisionnel de ces cartes est immédiat dans beaucoup de cas rencontrés au laboratoire ou dans des conditions industrielles.

De plus, les modèles d'écoulement devront pouvoir prédire les transitions ainsi mises en évidence : les résultats de l'étude des configurations pourra être un test sérieux des modèles d'écoulements élaborés dans d'autres cadres.

\section{Références citées}

[1] BONNIN J. - Rapport POO 76-3, EDF

[2] FITREMAN J.M., thèse de doctorat (1977), Université de Paris VI.

[3] JONES O. et ZUBER N. - Int. J. Multiphase Flow, Vol. 2 , 1975, pp. 273-306. 